\title{
ON THE THEOREMS OF Y. MIBU AND G. DEBS ON SEPARATE CONTINUITY
}

\author{
ZBIGNIEW PIOTROWSKI \\ Department of Mathematics \\ Youngstown State University \\ Youngstown, OH 44555 USA
}

(Received May 25, 1994 and in revised form November 1, 1995)

\begin{abstract}
Using a game-theoretic characterization of Baire spaces, conditions upon the domain and the range are given to ensure a "fat" set $C(f)$ of points of continuity in the sets of type $X \times\{y\}$, $y \in Y$ for certain almost separately continuous functions $f: X \times Y \rightarrow Z$ These results (especially Theorem B) generalize Mibu's First Theorem, previous theorems of the author, answers one of his problems as well as they are closely related to some other results of Debs [1] and Mibu [2]
\end{abstract}

KEY WORDS AND PHRASES: Separate and joint continuity, quasi-continuity, Moore spaces. 1991 AMS SUBJECT CLASSIFICATION CODES: 54C08, 54C30, 26B05

\section{INTRODUCTION}

Since the appearance of the celebrated result of I Namioka, many articles have been written on the topic of separate and joint continuity, see Piotrowski [3], for a survey

Aside from an intensively studied Uniformization Problem - Namioka-type theorems, see Piotrowski [3], questions pertaining to Existence Problem (see below) as well as its generalizations, have been asked

Let $X$ and $Y$ be "nice" (e g Polish) topological spaces, let $M$ be metric and let $f: X \times Y \rightarrow M$ be separately continuous, that is, continuous with respect to each variable while the other is fixed Find the set $C(f)$ of points of (joint) continuity of $f$.

Let us recall that given spaces $X, Y$ and $Z$, and let $f: X \times Y \rightarrow Z$ be a function. For every fixed

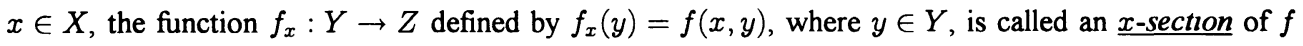
A $y$-section $f_{y}$ of $f$ is defined similarly.

One way to ensure the existence of "many" points of continuity in $X \times Y$ can be derived from the following. Baire-Lebesgue-Kuratowski-Montgomery Theorem (see Piotrowski [3]) Let $X$ and $Y$ be metric and let $f: X \times Y \rightarrow \mathbb{R}$ have all $x$-sections $f_{x}$ continuous and have all $y$-sections $f_{y}$ of Baire class $\alpha$ Then $f$ is of class $a+1$ 
If $\alpha=0$, that is, $f_{y}$ is continuous, $f$ is of class 1 Now, by a theorem of Baire, $C(f)$ is residual So, If we assume additionally that $X \times Y$ is Baire, then $C(f)$ is a dense $G_{\delta}$ subset of $X \times Y$

But one cannot relax the assumptions pertaining to the sections too much

EXAMPLE. Let $I=[0,1]$ and let $\mathbb{R}$ be the set of reals Put $D_{n}=\left\{(x, y): x=\frac{k}{2^{n}}, y=\frac{p}{2^{n}}\right.$, where $k$ and $p$ are all odd numbers between 0 and $\left.2^{n}\right\}$ Let $D=\cup_{n=1}^{\infty} D_{n} \quad$ It is easy to see that $\mathrm{Cl} D=I^{2}$ Now, let us define $f: I^{2} \rightarrow \mathbb{R}$ by $f(x, y)=1$, for $(x, y) \in D$ and $f(x, y)=0$ if $(x, y) \notin D$ All the $x$ sections $f_{x}$ and all the $y$-sections $f_{y}$ are of first class of Baire and $C(f)=\phi$

However, the following three important results hold

MIBU'S FIRST THEOREM (Mibu [2]) Let $X$ be first countable, $Y$ be Baire and such that $X \times Y$ is Baire Given a metric space $M$ If $f: X \times Y \rightarrow M$ is separately continuous, then $C(F)$ is a dense $G_{\delta}$ subset of $X \times Y$

MIBU'S SECOND THEOREM [2]. Let $X$ be second countable, $Y$ be Baire and such that $X \times Y$ is Baire Given a metric space $M$ If $f: X \times Y \rightarrow M$ has

a) all $x$-sections $f_{x}$ have their sets $D(f)$ of points of discontinuity of the first category and,

b) all $y$-sections $f_{y}$ are continuous

Then $C(f)$ is a dense, $G_{\delta}$ subset of $X \times Y$

- Following Debs [1], a function $f: X \rightarrow M$ is called first class if for every $\epsilon>0$, for every nonempty subset $A \subset X$, there is a nonempty set $U$, open in $A$, such that $\operatorname{diam}(f(U)) \leq \epsilon$.

DEBS' THEOREM [1] Let $X$ be first countable $Y$ be a special $\alpha$-favorable space (thus Baire), $X \times Y$ be Baire Given a metric space $M$ If $f: X \times Y \rightarrow M$ has:

a) all $x$-sections $f_{x}$ of first class - in the sense of Debs and,

b) all $y$-sections $f_{y}$ continuous

Then $C(f)$ is a dense $G_{\delta}$ subset of $X \times Y$

\section{QUASI-CONTINUITY ON PRODUCT SPACES}

A function $f: X \rightarrow Y$ is called quasi-contmuous at a point $x \in X$ if for each open sets $A \subset X$ and $H \subset f(X)$, where $x \in A$ and $f(x) \in H$, we have $A \cap I n t f^{-1}(H) \neq \emptyset \quad$ A function $f: X \rightarrow Y$ is called quast-continuous, if it is quasi-continuous at each point $x$ of $X$.

A function $f: X \times Y \rightarrow Z(X, Y, Z$ - arbitrary topological spaces) is said to be quasi-continuous at $(p, q) \in X \times Y$ with respect to the variable $y$, if for every neighborhood $N$ of $f(p, q)$ and for every neighborhood $U \times V$ of $(p, q)$, there exists a neighborhood $V^{\prime}$ of $q$, with $V^{\prime} \subset V$, and a nonempty open $U^{\prime} \subset U$, such that for all $(x, y) \in U^{\prime} \times V^{\prime}$ we have $f(x, y) \in N$. If $f$ is quasi-continuous with respect to the variable $y$ at each point of its domain, it will be called quasi-continuous with respect to $y$ The definition of a function $f$ that is quasi-continuous with respect to $x$ is quite similar. If $f$ is quasicontinuous with respect to $x$ and $y$, we say that $f$ is symmetrically quast-continuous.

One can easily show from the definitions that if $f$ is symmetrically quasi-continuous, then $f_{x}$ and $f_{y}$ are quasi-continuous for all $x \in X$ and $y \in Y$. The converse does not hold.

LEMMA (Piotrowski [4] Theorem 42). Let $X$ be a Baire space, $Y$ be first countable and $Z$ be regular If $f$ is a function on $X \times Y$ to $Z$ such that all its $x$-sections $f_{x}$ are continuous and all its $y$ sections $f_{y}$ are quasi-continuous, then $f$ is quasi-continuous with respect to $y$

The converse does not hold 
As an immediate consequence we obtain (Piotrowski [4] Corollary 43) Let $X$ and $Y$ be first countable, Baire spaces and $Z$ be a regular one If $f: X \times Y \rightarrow Y$ is separately continuous, then $f$ is symmetrically quasi-continuous

If $X$ and $Y$ are second countable Baire spaces and $Z$ is a regular one, and a function $f: X \times Y \rightarrow Z$, then the following implications hold (which show the inclusion relations between proper classes of functions) - see Diagram 1 None of these implications can, in general be replaced by an equivalence, see Neubrunn [5]

\begin{tabular}{|c|c|c|c|c|}
\hline \multicolumn{5}{|c|}{$f$-continuous } \\
\hline & 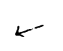 & $\downarrow$ & $>$ & \\
\hline$f$-symmetrically & $\leftarrow$ & $f_{x}, f_{y}$-continuous & $\rightarrow$ & $f$-quasi-continuous \\
\hline quasi-continuous & $\rightarrow$ & $\stackrel{\downarrow}{\downarrow} f_{y}$-quasi-continuol & $x$ & \\
\hline
\end{tabular}

Diagram 1

The Banach-Mazur game. We will use here the classical Banach-Mazur game between players $A$ and $B$ both playing with perfect information (see Noll [6], Oxtoby [7]) A strategy for player $A$ is a mapping $\alpha$ whose domain is the set of all decreasing sequences $\left(G_{1}, \ldots, G_{2 n-1}\right), n \geq 1$, of nonempty open sets such that $\alpha\left(G_{1}, \ldots, G_{2 n-1}\right)$ is a nonempty open set contained in $G_{2 n-1}$. Dually, a strategy for player $B$ is a mapping $\beta$ whose domain is the set of all decreasing sequences $\left(U_{1}, \ldots, U_{2 n}\right), n \geq 0$, of nonempty open sets such that $\beta\left(U_{1}, \ldots, U_{2 n}\right)$ is nonempty, open and contained in $U_{2 n}$ Here $n=0$ stands for the empty sequence, for which $\beta(\emptyset)$ is nonempty and open, too. If $\alpha, \beta$ are strategies for $A, B$ respectively, then the unique sequence $G_{1}, G_{2}, G_{3}, \ldots$ defined by $\beta(\emptyset)=G_{1}, \alpha\left(G_{1}\right)=G_{2}$, $\beta\left(G_{1}, G_{2}\right)=G_{3}, \alpha\left(G_{1}, G_{2}, G_{3}\right)=G_{4}, \ldots$ is called the game of $A$ with $\alpha$ against $B$ with $\beta$ We will say that $A$ with $\alpha$ wins against $B$ with $\beta$ if $\cap\left\{G_{n}: n \in N\right\} \neq \emptyset$ holds for the game $G_{1}, G_{2}, \ldots$ of $A$ with $\alpha$ against $B$ with $\beta$. Conversely, we will say that $B$ with $\beta$ wins against $A$ with $\alpha$ if $A$ with $\alpha$ does not win against $B$ with $\beta$.

We will make use of the following theorem, essentially proved by Banach and Mazur cf. Oxtoby [7], see also Noll [6] where the game-theoretic characterization of Baire spaces was applied to obtain some graph theorems.

Let $E$ be a topological space. The following are equivalent:

(1) $E$ is a Baire space;

(2) for every strategy $\beta$ of $B$ there exists a strategy $\alpha$ of $A$ with $\alpha$ wins against $B$ with $\beta$. 


\section{THE MAIN RESULT}

Let us recall If $A \subset X$ and $\mathcal{U}$ is a collection of subsets of $X$, then st. $(A, \mathcal{U})=\bigcup\{U \in \mathcal{U}$.

$U \cap A \neq \emptyset\}$ For $x \in X$, we write $s t .(x, \mathcal{U})$ instead of st. $(\{x\}, \mathcal{U})$ A sequence $\left\{G_{n}\right\}$ of open covers of $X$ is a development of $X$ if for each $x \in X$ the set $\left\{\right.$ st. $\left.\left(x, G_{n}\right): n \in N\right\}$ is a base at $x$ A developable space is a space which has a development A Moore space is a regular developable space

THEOREM A. Let $X$ be a Baire space, $Y$ be space and let $\left\{P_{n}\right\}_{n_{-1}}^{x_{+}}$be a development for $Z$ If $f: X \times Y \rightarrow Z$ is quasi-continuous with respect to $y$, then $C(f)$ is a dense, $G_{\ell}$ subset in $X \times\{y\}$, for all $y \in Y$

PROOF. Let $x \in X, y \in Y$ and let $U \times V$ be a neighborhood of $(x, y)$ Define a strategy $\beta$ for a player $B$ in a corresponding Banach-Mazur game played over $X$ For this purpose we shall order (wellordering) the sets $X$, open neighborhoods of $y$ and open nonempty subsets of $X$

(1) $\beta(\emptyset)$ has to be defined Since $Z$ has a countable development $\mathcal{P}_{n}$, there is a local countable base at every point of $Z$, in particular take $\left\{G_{n}\right\}$ at $f(x, y)$ Pick $G_{1}$ Now by the quasi-continuity of $f$ with respect to $y$, there is a neighborhood $V^{1}$ of $y$, and a nonempty open $U^{1}$ such that $f\left(U^{1} \times V^{1}\right) \subset G_{1} \quad$ Let us further assume that $U^{1}$ and $V^{1}$ are the first sets in their orderings of $X$ and $Y$, respectively with the above property Now, let $W^{1}$ be the first nonempty open set contained in $U^{1}$ and let $x_{1}$ be the first element of $W^{1}$ Thus, $W^{1} \times V^{1}$ is a neighborhood of $\left(x_{1}, y\right)$ So, let $\beta(\emptyset)=W^{1}$

(2) $\beta\left(G_{1}, G_{2}\right)$ has to be defined, where $G_{1}, G_{2}$ are nonempty open and $G_{2} \subset G_{1}$ Now, $f$ is quasicontinuous with respect to $y$ at $\left(x_{2}, y\right)$, pick $G^{3}$, the first element of the base at $f\left(x_{1}, y\right)$ with $G_{3} \subset G_{2}$ Now pick the first element $U^{3} \times V^{3}$ such that $f\left(U^{3} \times V^{3}\right) \subset G_{3}$ - such a $U^{3} \times V^{3}$ exists, by the quasi-continuity with respect to $y$ of $f$ Now, let $W^{3}$ be the first open nonempty set contained in $U^{3}$ (a priori, it can be even the same set (!)) and let $x_{3} \in U^{3}$ be the first element of $W^{3}$. Thus, $W^{3} \times V^{3}$ is a neighborhood of $\left(x_{3}, y\right)$ So, let $\beta\left(G_{1}, G_{2}\right)=W^{3}$.

(3) In this way we proceed to define $\beta$ by recursion, i e., if $\beta(\emptyset)=G_{1}$ and $\beta\left(G_{1}, \ldots, G_{2 k}\right)=G_{2 k+1}$, for all $k<n$ then the former steps are available and we can define $G_{2 k+1}$ in analogy with (2).

(4) Suppose now that $\beta$ has been defined. Since $X$ is Baire, there is a strategy $\alpha$ for $A$ such that $A$ with $\alpha$ wins against $B$ with $\beta$ (see the definition of the game).

Let $G_{1}, G_{2} \ldots$ be the game $A$ with $\alpha$ against $B$ with $\beta$.

Notice that.

$$
\bigcap\left\{W_{n}: n \in N\right\}=\bigcap\left\{G_{n}: n \in N\right\} \text {. }
$$

But observe that $\alpha$ is winning, hence this intersection is nonempty; i.e, $x^{*} \in \bigcap\left\{W_{n}: n \in N\right\}$, so $\left(x^{*}, y\right) \in(U \times V) \cup(X \times\{y\})$. This in turn shows the density of $C(f)$ in $X \times\{y\}$ The $G_{\delta}$ part follows easily from the construction

A space will be called quast-regular if for every nonempty open set $U$, there is a nonempty open set $V$ such that $\mathrm{Cl} V \subset U$ Obviously, every regular space is quasi-regular.

Let $\mathcal{A}$ be an open covering of a space $X$ Then a subset $S$ of $X$ is said to be $\mathcal{A}$-small if $S$ is contained in a member of $\mathcal{A}$ A space $X$ is said to be strongly countably complete if there is a sequence 
$\left\{A_{1} \cdot \imath=1,2, \ldots\right\}$ of open coverings of $X$ such that a sequence $\left\{F_{1}\right\}$ of closed subsets of $X$ has a nonempty intersection provided that $F_{,} \supset F_{l, l}$ for all $\imath$ and each $F$, is $\mathcal{A}_{\iota}$-small

The class of strongly countably complete spaces includes locally countable compact spaces and complete metric spaces

In view of the following (Piotrowski [8], Theorem 46, see also Lemma 3 of Piotrowskı [9]) Every quasi-regular, strongly countably complete space $X$ is a Baire space

Theorem $\mathrm{A}$ is a strong generalization of the following

(Piotrowski [8], Theorem 45) Let $X$ be a space, $Y$ be quasi-regular, strongly countably complete and $Z$ be metric If $f: X \times Y \rightarrow Z$ is quasi-continuous with respect to $x$, then for all $x \in X$ the set of points of joint continuity of $f$ is a dense $G_{\delta}$ of $\{x\} \times Y$ Further, observe that our Theorem A answers, in positive, the following

(Piotrowski [8], Problem 4 11) Does Theorem 45 (of [8]) hold if $Y$ is only assumed to be a quasiregular Baire space?

The following Theorem B is the main result of this paper and its proof easily follows from the lemma and Theorem A

THEOREM B. Let $X$ be first countable, $Y$ be Baire and $Z$ be Moore If $f: X \times Y \rightarrow Z$ has all its $x$-sections $f_{x}$ quasi-continuous and all its $y$-sections $f_{y}$ continuous, then for all $x \in X$, the set of points of continuity of $f$ is a dense $G_{\delta}$ subset of $\{x\} \times Y$

The above result strongly generalizes (see the assumptions upon $Y$ and $Z$ ) the following known theorem

(Piotrowski [8], Theorem 48, see also Theorem 5 of Piotrowski [9]) Let $X$ be first countable, $Y$ be strongly countably complete, quasi-regular and $Z$ be a metric space If $f: X \times Y \rightarrow Z$ is a function such that all its $x$-sections $f_{x}$ are quasi-continuous and all its $y$-sections $f_{y}$ are continuous, then for all $x \in X$, the set of points of joint continuity of $f$ is a dense $G_{\delta}$ subset of $\{x\} \times Y$

Our Theorem B generalizes in many ways Mibu's First Theorem - see Introduction

It is also closely related to Mibu's Second Theorem and Debs' Theorem - ibidem Observe though, that quasi-continuity of a function does not imply - nor is implied, by the condition of being of first class - in the sense of Debs

Really, let $f:[0,1] \rightarrow \mathbb{R}$ be given by $f(x)=0$, if $x \neq \frac{1}{2}$. Then such a function $f$ is of first class, in the sense of Debs and, clearly, it is not quasi-continuous

There are quasi-continuous functions $f: \mathbb{R} \rightarrow \mathbb{R}$ which are of arbitrary class of Baire or not Lebesgue measurable - see Neubrunn [5] for more details

REMARK 1. The studies of the continuity points of functions whose ranges are not necessarily metric have been done already in the 1960's, see Klee and Schwarz [10] or later in the 1980's, see Dubins [11], we omit here an extensive literature of this approach, when the range is a uniform space

REMARK 2. Recently, the author has obtained some results of this paper using though entirely different techniques, see Piotrowski [12]

ACKNOWLEDGMENT. The author wishes to express his gratitude to a referee whose comments and remarks have improved the presentation of the results Also, thanks are due to the Research Council of Youngstown State University for a grant which enabled the author to complete this research 


\section{REFERENCES}

[1] DEBS, G, Fonctions separément continues et de premiere classe sur espace produit, Math. Scand. 59 (1986), 122-130

[2] MIBU, Y, On quasi-continuous mappings defined on product spaces, Proc. Japan Acad. 192 (1958), 189-192

[3] PIOTROWSKI, Z, Separate and joint continuity, Real Analysis Exchange, 11 (1985), 293-322

[4] PIOTROWSKI, Z, Quasi-continuity and product spaces, Proc. Intern. ('onf. Geom. Top., Warsawa (1980), 349-352

[5] NEUBRUNN, T, Quasi-continuity, Real Analysts Exchange, 14 (1988-89), 259-306

[6] NOLL, D , Baire spaces and graph theorems, Proc. Amer. Math. Soc., 96 (1986), 141-151

[7] OXTOBY, JC, The Banach-Mazur game and Banach category theorem, Contribution to the Theory of Games, Vol 3, Ann. of Math. Studies, No 39, Princeton Univ Press, Princeton, NJ, 1957

[8] PIOTROWSKI, Z, A study of certain classes of almost continuous functions on topological spaces, $\mathrm{Ph}$ D. Thesis, Wroclaw, 1979

[9] PIOTROWSKI, Z, Separate almost continuity and joint continuity, Colloquia Mathematica János Bolyal 23 Topology, Budapest (Hungary) (1978), 957-962

[10] KLEE, V, Stability of the fixed point property, Colloq. Math. 8 (1961), 43-46.

[11] DUBINS, L E and SCHWARZ, G., Equidiscontinuity of Borsuk-Ulam functions, Pacific J. Math., 95 (1981), 51-59

[12] PIOTROWSKI, Z, Mibu-type theorems, Classical Analysis, Proc. Intern. Conf. WSI, Radom (1994), 133-139 


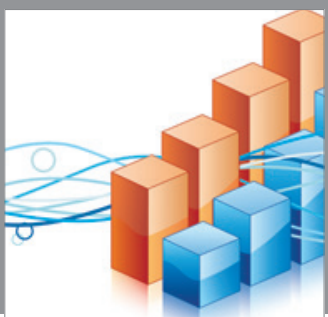

Advances in

Operations Research

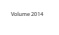

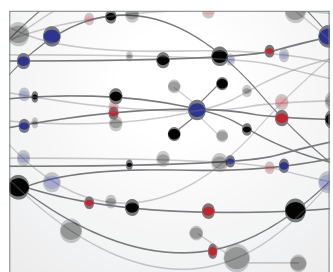

\section{The Scientific} World Journal
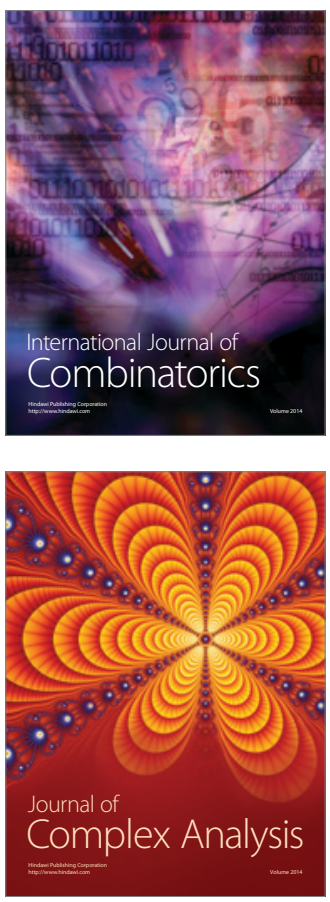

International Journal of

Mathematics and

Mathematical

Sciences
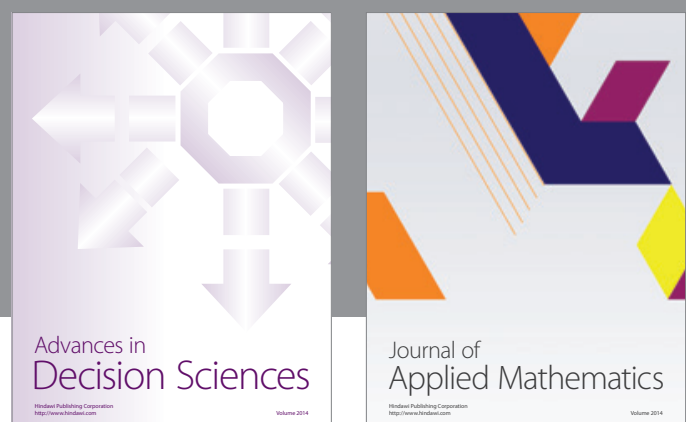

Journal of

Applied Mathematics
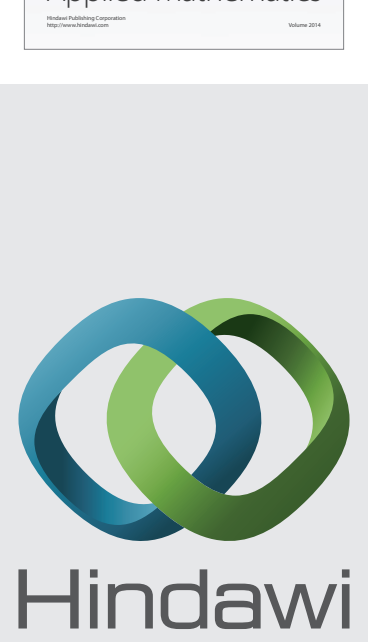

Submit your manuscripts at http://www.hindawi.com
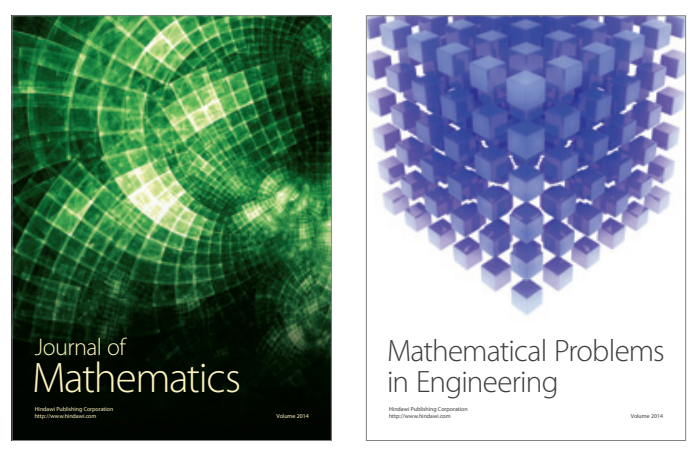

Mathematical Problems in Engineering
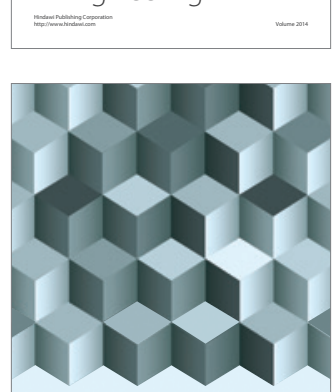

Journal of

Function Spaces
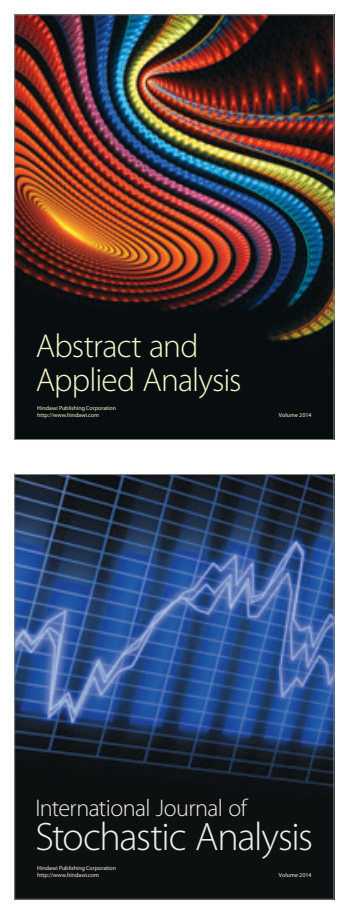

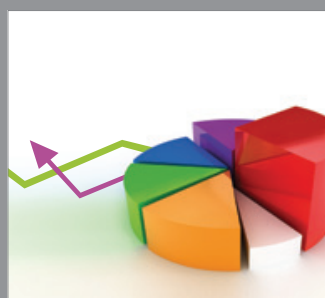

ournal of

Probability and Statistics

Promensencen
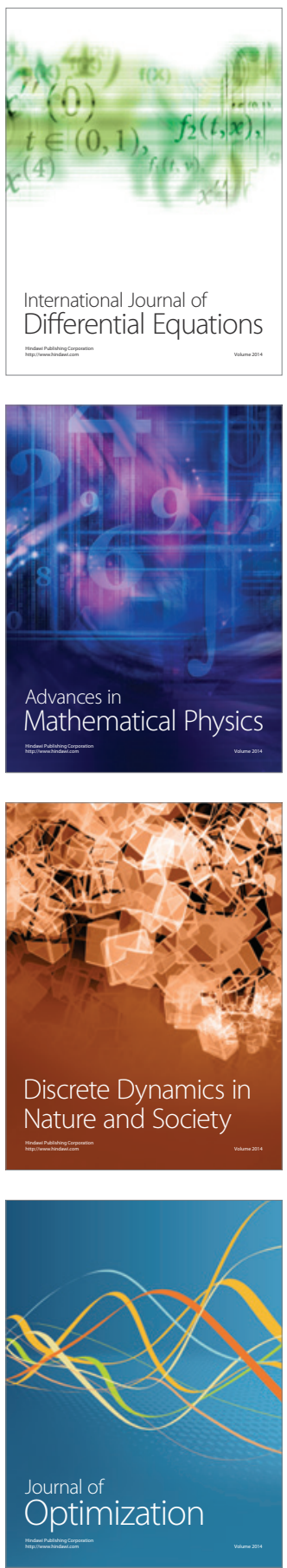